\title{
Top quark reconstruction in ATLAS
}

\author{
Vadim Kostyukhin* \\ INFN Genova (Italy) \\ E-mail: vadim.kostyukhin@cern.ch
}

ATLAS detector with powerful calorimetry and precise tracking system has the excellent capabilities to investigate the top quark sector of SM. To exploit fully an ATLAS potential and benefit from high LHC top quark production cross section, one needs to define a reconstruction strategy for accessing top quark physics in a most precise way. Jet finding, jet energy calibration and jet tagging in ATLAS will be described, together with constraint fits for restoring top quark kinematics parameters. Systematic errors introduced by reconstruction will be discussed. Quick top physics measurements during ATLAS detector commissioning will also be considered.

International Workshop on Top Quark Physics

January 12-15, 2006

Coimbra, Portugal

${ }^{*}$ Speaker. 


\section{Introduction}

At the LHC, the top quark will be produced mainly in pairs through the hard process $g g \rightarrow t \bar{t}$ (90\% of the total $t \bar{t}$ cross-section) and $q \bar{q} \rightarrow t \bar{t}$ (remaining $10 \%$ of the cross-section). The nextto-leading order cross-section prediction for $t \bar{t}$ production is $\sigma(t \bar{t})=833 \mathrm{pb}$ [1]. Thus the LHC will be a top factory as more than 8 million $t \bar{t}$ pairs will be produced per year at low luminosity (corresponding to an integrated luminosity of $10 \mathrm{fb}^{-1}$ ). The electroweak single top production processes, whose cross-sections are in total approximately one third of those of $t \bar{t}$ production, give additionally more than 3 millions of top quarks.

Even taking into account the process selection efficiency the remaining statistics for many physics measurements is big and doesn't limit a precision of the measurements. Then final accuracy of many LHC top physics results will be limited mainly by systematics effects in top quark selection and reconstruction. For example as it was shown in [1,2] after one year of data taking at low luminosity the statistical precision on top quark mass measurement will be better than $100 \mathrm{MeV}$ whereas the systematic error will be of the order or bigger than $1 \mathrm{GeV}$.

To exploit fully an ATLAS top-physics potential one needs to identify all sources of systematic errors in reconstruction chain and to use special approaches to keep them under control. Below I'll try to present ATLAS efforts in this direction.

\section{Jet reconstruction}
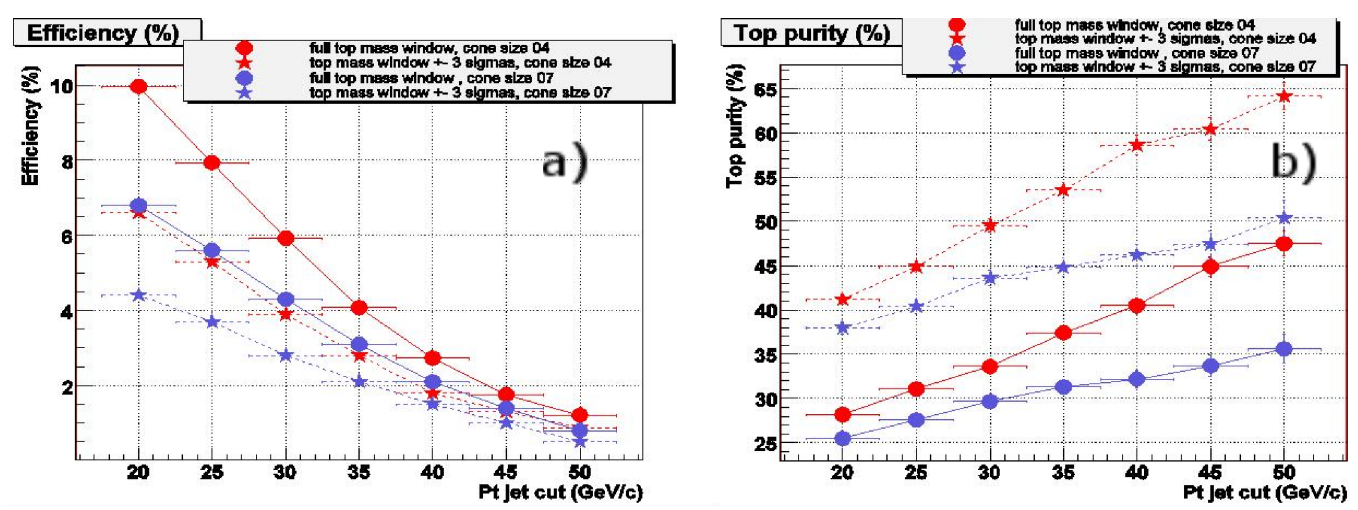

Figure 1: Efficiency and purity of top quark reconstruction as a function of jet $P_{t}$ cut for different $\Delta R$-cone sizes used in the jet finding algorithm. Red circles and stars - cone $\Delta R=0.4$ for complete mass scale and top mass peak window correspondingly. Blue circles and stars - the same for cone $\Delta R=0.7$.

Key issue for many top studies is a jet reconstruction. Up to now 3 methods have been used intensively for top quark reconstruction studies in ATLAS

1. Cone $\Delta R=0.4$

2. Cone $\Delta R=0.7$

3. $K_{t}$ with $d=1$ 
Scale factor $\mathrm{d}=1$ for $K_{t}$ algorithm makes it similar to cone algorithm with $\Delta R=0.7$.

Full simulation [3] of "lepton+jets" $t \bar{t} \rightarrow j j b b l v$ channel was used to verify an efficiency and accuracy of these jet algorithms. It was demonstrated that cone type algorithm with $\Delta R=0.4$ provides better purity of reconstructed sample and bigger top quark reconstruction efficiency (see fig.1.

Another important feature of any jet algorithm is a precision of underlying parton direction reconstruction especially in dense jet environment like $t \bar{t}$. This precision affects both b-tagging and accuracy of kinematical reconstruction. The angular accuracy of different jet algorithms is illustrated on fig. 2 where a minimal distance between b-quark and nearest jet in $t \bar{t}$ events is shown.

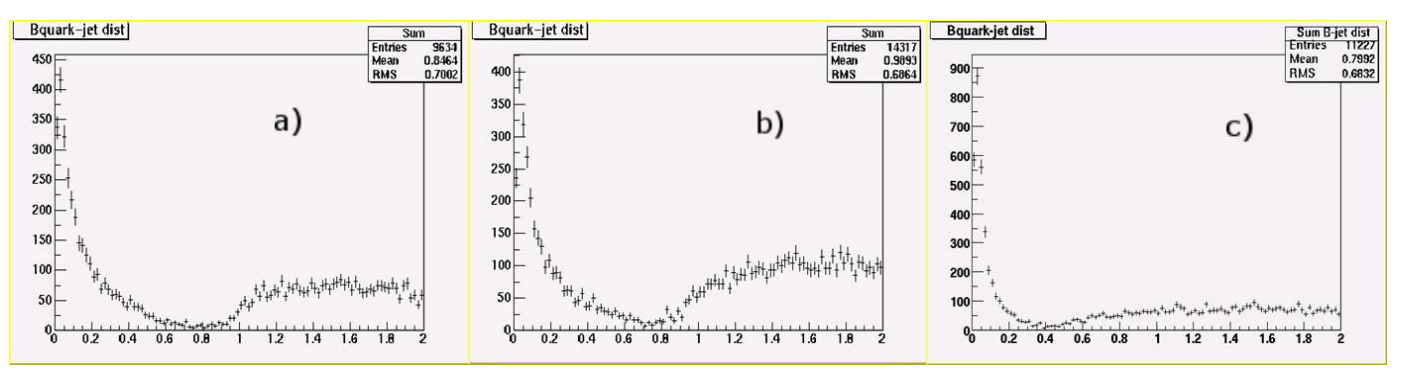

Figure 2: b-quark-Jet angular distance for different jet algorithms in $t \bar{t}$ events. a) - $K_{t}$ algorithm with $d=1$, b) - Cone algorithm with $\Delta R=0.7, \mathrm{c}$ ) - Cone algorithm with $\Delta R=0.4$

Cone jet reconstruct ruction algorithm with $\Delta R=0.4$ seems the best choice for top quark reconstruction. Another possibility could be a $K_{t}$ algorithm with scale factor $d=0.5$.

\section{Jet calibration}

Top quark properties can be measured based on distributions of top decay products. But top decay products are partons and leptons, and not jets. Unfortunately any detector is able to reconstruct only jets. So one needs to estimate parton kinematical parameters based on jet kinematical parameters. This step is generally a source of many systematic errors.

The most evident problem here is a parton energy estimation based on energy detected in calorimeter. This problem can be divided into two steps:

1. Detector based corrections

2. Physics based corrections

Detectors based corrections take into account noncompensation of calorimeter, longitudinal energy leakage, detector noise, cracks and nonuniformity of response, dead material, etc. The purpose of application of these correction is to have an unbiased estimation of energy of all stable particles hitting the given calorimeter region. Usually detector based corrections are rather straightforward, although they require a lot of technical work like dead material estimation, detector weighing, nonlinearity corrections, MC tuning, single particle calibration, etc.

Physics based corrections deal with energy leak outside jet cone, pileup, final state radiation, leptonic decays, etc. The purpose of this step is to estimate the direction and energy of underlying 
parton based on stable particles energy flow (obtained with calorimeter). Some of the physics corrections are simple (pileup), but the others are completely non-trivial and in many cases MC based only. Very often they are also process and reconstruction algorithm dependent. This makes a task of identification of all systematic errors introduced at this stage very complicated. Below some of systematic errors present at physics correction stage will be presented.

\subsection{Semyleptonic B decays}

Inside b-jets many B and D mesons decay semyleptonically. Undetectable neutrinos from these decays take away part of jet energy and this part is random. So semyleptonic decays in b-jets result in

- Worsening of b-jet energy resolution (additional to calorimeter performance)

- Nongaussian and cut dependent shapes of all kinematical distributions with b-jets

Simple taking into account a mean energy loss for b-jets due to semyleptonic decays is able to restore a mean jet energy, but there is no way to restore a normal (calorimeter) jet energy resolution and there is no way to correct non-gaussian shape of "true jet"-"reconstructed jet" energy distribution.
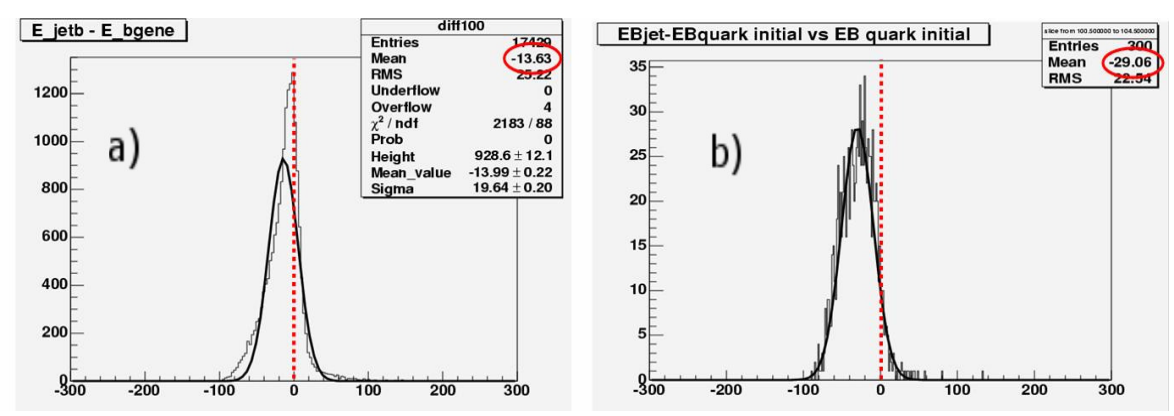

Figure 3: b-quark-Jet angular distance for different jet algorithms in $t \bar{t}$

Taking into account the big cross-section of top quark production at LHC it seems possible not to use for analysis the b-jets where neutrino is produced in heavy $(B, D)$ meson decay. Such jets can be detected by presence of lepton inside them. The actual loss of statistics in this case will depend significantly on efficiency of lepton in jet detection and may reach $50 \%$. But for example for top quark mass measurement at LHC where statistics is more than enough, the removing of b-jets with lepton inside may really improve systematic errors.

\subsection{Jet mass}

For top quark mass estimation and for measurements of angular correlations in top decays the quark parameters should be used in ideal case. In practice any detector gives jet parameters. Let's assume for the moment that jet energy problem is somehow solved t.e. jet is calibrated. Then how well jet reproduce the parton direction? 
There is a principal difference between jet and parton. Parton is a single (pointlike) particle. Jet is a collection of particles. Lorentz boost produces different results when applied to single particle or particle collection. As an example one may consider a $\mathrm{W}$ decay. In $\mathrm{W}$ center of mass frame system it's possible to use either two back-to-back partons or two back-to-back jets with corresponding energy normalization. There is no difference between two different descriptions for kinematical calculations. But after translation to laboratory frame system the angle between 2 partons and angle between 2 jet axes ${ }^{1}$ will be different. This is illustrated in fig. 4 .


Figure 4: Decay of the massive particle $M$ to massless partons and massive jets in center of mass frame and laboratory frame.

This simple observation has several consequences. First of all in the laboratory reference frame jet direction has a systematic shift with respect to underlying parton direction and this shift is independent on jet reconstruction algorithm. Then any mass based (W,Z) jet energy calibration is different from $P_{t}$ based (di-jets, Z+jets) calibration in usual massless jet approach due to systematic shift of jet-jet opening angle. All differences are at a few percent level ( for situation in fig.4 $\frac{\phi_{2}}{\phi_{1}} \approx 1-\frac{m^{2}}{M^{2}} \approx 2 \%$ for $\mathrm{m}=8 \mathrm{GeV}$ and $\mathrm{M}=80 \mathrm{GeV}$ ) but on LHC $1 \%$ shift of jet energy scale produce the $0.7 \mathrm{GeV}$ shift in top quark mass estimation.

A natural way to take into account the jet-parton difference in kinematical calculations with jets is to introduce jet masses. Any particle collection like jet has the invariant mass. The jet mass is not a well defined variable because it has a strong jet reconstruction algorithm dependence, but it allows to compensate a big part of the effect. Massive jet approach has lower systematic in all kinematical estimations in comparison with massless jet approach. For massive jets the $P_{t}$ based and mass based calibration must give the same results.

\subsection{Statistical systematics in jet energy calibration}

A natural way to calibrate jet energy for top physics is a $\mathrm{W}$ mass peak in $t \bar{t}$ events. Jet energy correction function can be obtained through $\mathrm{W}$ peak position shift for different jet energies $R=\frac{M_{W}^{P D G}}{M_{W}^{f i t}}=\sqrt{\alpha_{1} \cdot \alpha_{2}}$. Due to a huge $t \bar{t}$ statistics this calibration potentially is very precise.

A standard way to do this calibration is a histogram approach (binning). Either $E_{j e t}\left(E_{\text {parton }}\right)$ or $E_{\text {parton }}\left(E_{j e t}\right)$ distributions are histogrammed and then parametrized. Theoretically both parametrizations must give the same results because any of these functions is just the inverse of another one. In practice the results are different. The difference is demonstrated in fig. 5 where the ratio $\frac{E_{\text {jer }}^{\text {corrected }}}{E_{\text {parton }}}$ is shown as a function of $E_{\text {jet }}$ and $E_{\text {parton }}$. For calculation of the $E_{\text {jet }}^{\text {corrected }}$ in both cases the $E_{j e t}\left(E_{\text {parton }}\right)$ parametrization was used.

\footnotetext{
${ }^{1}$ jet axis is determined as an averaged direction of particle collection
} 

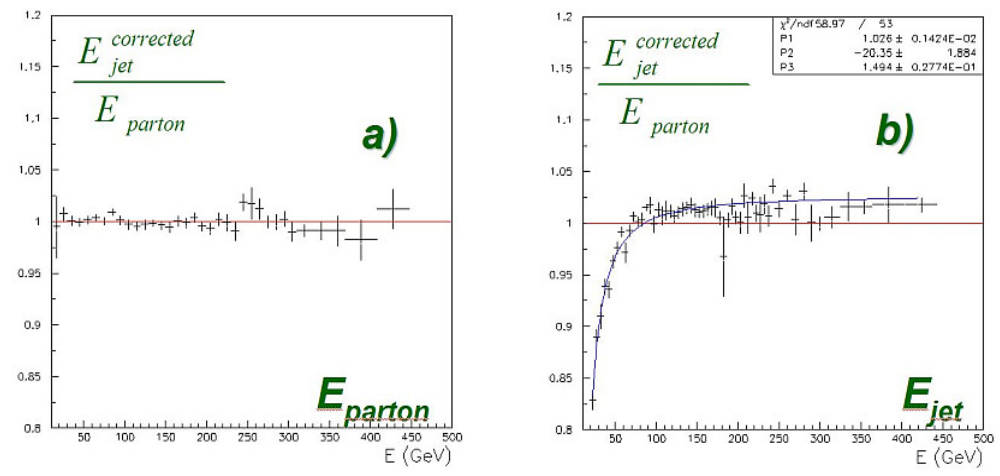

Figure 5: Ratio $\frac{E_{\text {jet }}^{\text {corected }}}{E_{\text {parton }}}$ obtained with $E_{\text {jet }}\left(E_{\text {parton }}\right)$ parametrization. a) - the ratio is shown as function of $\left.E_{\text {parton }}, \mathrm{b}\right)$ - the ratio is shown as function of $E_{\text {jet }}$.

The observed dependence on parametrization choice is mainly due to a nonconstant and nonlinear jet energy resolution. Together with a nontrivial jet energy spectrum in $t \bar{t}$ events it results in very complex leaks between different bins in calibration distributions. As a consequence the $E_{\text {jet }}\left(E_{\text {parton }}\right)$ and $E_{\text {parton }}\left(E_{\text {jet }}\right)$ parametrizations are not the inverse of each other. Strictly speaking none of the calibration function obtained with simple binning method is correct. And a flatness of distribution on fig.5a) is not a guaranty that kinematical distributions obtained with $\left(E_{\text {jet }}^{\text {corrected }}\right.$ are not systematically shifted.

On LHC in many cases jet energy calibration must be precise at $1 \%$ (and better...) level. A simple binning approach is not able to provide this level of accuracy. Special function deconvolution methods are needed to guarantee the systematic precision in jet energy calibration at $1 \%$ level.

\section{B-tagging}

For lifetime based b-tagging ATLAS is developing different types of algorithms. All of them use track impact parameters of tracks in jet, but they follow different approaches in making a $b / n o n-b$ decision for the jet based on track information. One may mention a simple track counting, secondary vertex detection, probabilistic[4] approach, and LogL[5] approach. The most powerful ATLAS tagger is based on LogL approach and is a combination of track based and secondary vertex in jet based likelihoods.

$$
W_{\text {jet }}=W_{\text {tracks }}+W_{\text {sec.vertex }}=\sum_{i=1}^{N_{\text {track }}} \log \frac{b\left(S_{i}\right)}{u\left(S_{i}\right)}+\log \frac{b(M, F, N)}{u(M, F, N)}
$$

here $W_{\text {jet }}$ is a weight variable used for b-jet selection, $b(\ldots)$ and $u(\ldots)$ are the distribution functions for b-jets and light quark jets correspondingly. Performance of the combined algorithm is quite satisfactory and is limited mainly by physics factors (gluon splitting into $b \bar{b}$ pair and jet overlaps). In special "purified" conditions in $t \bar{t} j j$ events with 6 jets in final states the b-tagging light jet rejection may reach 2500 at $50 \%$ b-tagging efficiency. A typical example of the light jet 
rejection as a function of b-jet tagging efficiency is shown in fig.6 for different tagging algorithm and nonpurified events.

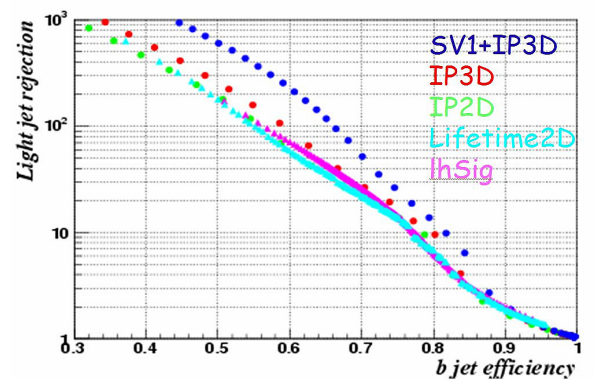

Figure 6: B-tagging light jet rejection as function of tagging efficiency in "lepton+jets" $t \bar{t} \rightarrow l v j j b b$ channel for different tagging algorithms

In spite of intuitively clear definition of "b-jet" and "light quark jet" the interpretation of btagging results is not straightforward. On MC data usually "b-jet" and "light quark" jets are labeled based on jet-parton distance. Even in this case the b-tagging performance depends on cuts used during labeling.

For example one may label a jet as a light quark one if distance between this jet and the nearest b-quark is bigger than 0.3. In this case a rejection of one of the ATLAS b-taggers(SV1) will be 184 and $60 \%$ efficiency. But if a definition of light quark jet is changed so that a minimal distance with the nearest b-quark is 0.8 , then the same rejection will be 240 at $60 \%$ efficiency. So any definition of b-tagging efficiency and rejection is umbiguous even on MC data if the jet-parton distance is used for jet labeling. And at last there is no any jet-parton distance on real data, what makes impossible the MC-data comparison.

The only way out is to use for b-tagging performance validation and data-MC comparison the "single jet" events, or events with well isolated jets, which have a priory known type (without any jet-parton distance) like di-jet events or $t \bar{t} \rightarrow l v l v b b$ events. Only in this case the jet type and then b-tagging performance are unambiguous and then b-tagging MC may be tuned on real data. The multijet events like $t \bar{t} \rightarrow l v j j b b$ don't allow a systematic free MC-data comparison and tuning due to the problems with understanding what is a "b-jet" there.

For physics measurements the b-tagging efficiency and light jet rejection are not primary. Primary values are the "event selection efficiency" and "background rejection efficiency" with btagging. Even having a precise and well tuned on data estimation of b-tagging performance on single isolated jet, the propagation of this performance to multijet environment is not straightforward. Jets can overlap, performance of any jet reconstruction algorithm depends on jet density, $\alpha_{s}$ is process type dependent, etc... Any of these reasons and many others affect b-tagging performance in multijet events. So the only way to estimate correctly the event selection efficiency with b-tagging in multijet events is a correct MC. There is no way to predict with good accuracy the b-tagging performance in multijet events by using the single jet performance only.

It seems that the only systematic free at the level required for top quark physics at LHC way to work with b-tagging is a tuning of b-tagging software on "single jet" events (di-jet or $t \bar{t} \rightarrow$ 
$l v l v b b)$ and obtaining the required event selection and background rejection efficiencies with MC simulation of corresponding processes.

\section{Kinematical fit with constraints}

Kinematical fit with constraints is a useful tool for top quark reconstruction because it allows to improve a resolution of kinematical measurement measurements by using a priory information. For $t \bar{t}$ system there are 3 possible constraints if top quark mass is considered unknown, and 4 constraints if top mass is considered known. They are two W-mass constraints in two top quark decays, and $M_{t}=M_{\bar{t}}$ for unknown top mass or two top mass constraints for known top mass. The constraints are applicable because detector resolution (ATLAS case) is worse than the widths of both $\mathrm{W}$ and top quark decays.

One of the problems where kinematical fit with constraints is particularly important is a top quark mass measurement in "lepton + jets" channel $t \bar{t} \rightarrow l v j j b b$. One may easily show that from

$$
\left\{\begin{array}{cl}
(b+l+v)^{2} & =X_{t o p}^{2}=M_{W}^{2}+2 \cdot(b, l+v) \\
(l+v)^{2} & =M_{W}^{2}=2 \cdot(l, v)
\end{array}\right.
$$

where $X_{t o p}$ is a mass given by hadronic decay of second top in event, it follows

$$
\left\{\begin{array}{l}
E_{v} \cdot\left(E_{b}-p_{b} \cdot \cos \left(\phi_{b v}\right)\right)=\frac{X_{t o p}^{2}-M_{W}^{2}}{2}-(b, l) \\
E_{v} \cdot\left(E_{l}-p_{l} \cdot \cos \left(\phi_{l v}\right)\right)=\frac{M_{W}^{2}}{2}
\end{array}\right.
$$

$\mathrm{W}$ mass constraint defines only one angle between neutrino momentum and lepton $\phi_{l v}$ what gives an ambiguous neutrino direction. Second constraint (top mass) defines another angle of neutrino with respect to b-jet $\phi_{b v}$ what makes neutrino direction nonambiguous.
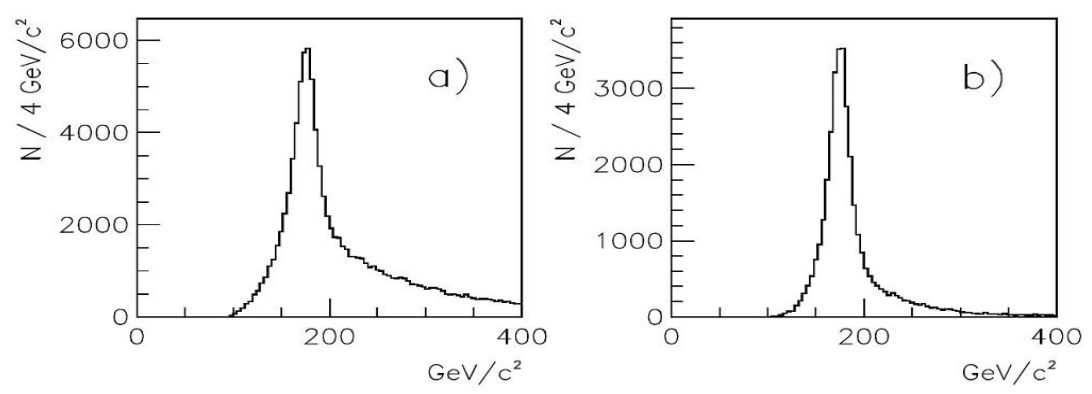

Figure 7: Result of kinematical fit with constraints application for top mass measurement in $t \bar{t} \rightarrow l v j j b b$ channel. a) - mass peak before constraint fit, b) - mass peak after constraint fit and $\chi^{2}$ based selection of correct jet assignment.

Additional benefit of kinematical constraint fit is a $\chi^{2}$ information after it. Based on $\chi^{2}$ it's possible to improve significantly a physical background rejection and to get rid of combinatorial background (wrong assignment of jets in top decay) because the background events have much bigger $\chi^{2}$ usually. The results of application of the constraint fit for $t \bar{t} \rightarrow l v j j b b$ are shown in 
fig.7. Due to the presence of neutrino in top decay an increase in resolution is not big, but a significant reduction of combinatorial background is easily achieved.

A simplest way to include constraints into fitting functional is to use a " $\chi^{2 "-t y p e ~ t e r m ~ l i k e ~}$ $\delta \chi^{2}=\frac{\left(M_{j j}-M_{W}\right)^{2}}{\Gamma_{W}^{2}}$ for $\mathrm{W}$ mass constraint. Such term provides slightly better resolution but it's not very robust and works well only for ideal jet calibration. Contrary to it a real constraint of the type $M_{j j}=M_{W}$ is significantly less sensitive to the systematic errors in jet energy calibration. Also the $M_{j j}=M_{W}$ constraint doesn't worsen appreciably the accuracy of the fit. It seems that for systematic-free kinematical measurements the exact constraints should be used and not the " $\chi^{2 "}$ type terms.

\section{ATLAS start-up}

Due to a very big cross-section of $t \bar{t}$ production even in a few first days of LHC running a sizable amount of top quark events will be obtained. Is it possible to use these events for initial cross-section and mass measurement and detector calibration?

According to the present ATLAS detector construction and commissioning plan at the time of the first LHC collisions detector will be as follow:

- Tracking and muon systems are not aligned

- Hadron calorimeter response is uniform due to Cs source calibration system, but not correctly scaled

- LAr electromagnetic calorimeter response is known with a few percent precision

- Trigger thresholds are increased to reduce rates

Then initial ATLAS detector will be able to reconstruct jets with good resolution but unknown energy scale. Also it will be able to detect high $P_{t}$ leptons (muons and probably electrons), but with extremely bad momentum estimations. B-tagging most probably will be absent due to the absence of the reasonable tracking.



Figure 8: Top quark and $\mathrm{W}$ mass peaks obtained with a simple $P_{t}$ based selection of jet

These reduced ATLAS capabilities nevertheless seem enough for selection of $t \bar{t} \rightarrow l v j j b b$ process which has $250 \mathrm{pb}$ production cross section. To do this one needs to require a presence of 4 
jets above some threshold and high $P_{t}$ lepton. With a reasonable choice of cuts ( $P_{t}^{\text {lepton }}>20 \mathrm{GeV}$, $P_{t}^{j e t}>40 \mathrm{GeV}$ ) a selection efficiency is $4.5 \%[6]$ what gives $11 \mathrm{pb}$ after selection. This cross section value is very approximate because of unknown energy scale for initial ATLAS, but it gives an order of magnitude for initial top statistics ( $1100 \mathrm{ev}$ for $100 \mathrm{pb}^{-1}$ at a first few days of running). Jet energy scale may be estimated by using $\mathrm{W}$ mass peak in top events themselves.

Top quark mass peak may be obtained[6] by simply selecting of 3 jets with highest $\vec{P}_{t}=$ $\sum_{i=1}^{3} \vec{P}_{t}^{j e t}$. Then a selection of 2 jets with highest $\vec{P}_{t}=\sum_{i=1}^{2} \vec{P}_{t}^{j e t}$ out of already selected 3 jets produces a nice peak of $\mathrm{W}$ decay (see fig.8).

The nice peaks on fig. 8 can be seen only if a background level is not very big. Main background in this case is a $\mathrm{W}+4$ jets process $[1,6]$. An estimation of the production cross section with an Alpgen generator and the same cuts as have been used for $t \bar{t}$ selection for $150 \mathrm{pb}^{-1}$ luminosity gives the result presented on fig.9. As a conclusion one may say that even with the reduced capabilities of initial ATLAS detector top quark signal is detectable in a first few days of LHC running.

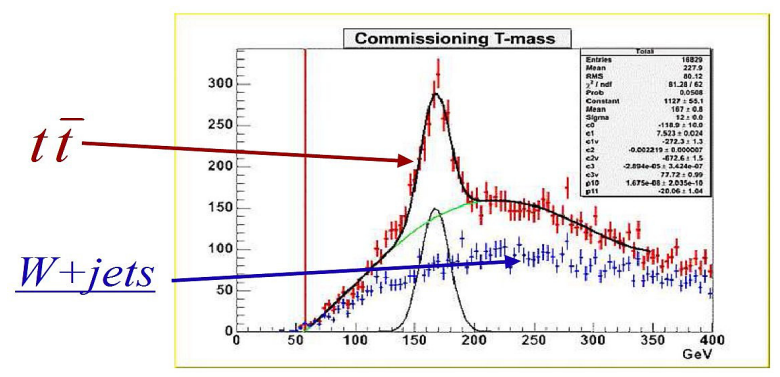

Figure 9: Top quark and $\mathrm{W}+4$ jets background with $150 \mathrm{pb}^{-1}$ luminosity

\section{References}

[1] ATLAS collaboration, "ATLAS Detector and Physics Performance : Technical Design Report", CERN-LHCC-99-015, 1999

[2] Borjanovic, I et al., "Investigation of top mass measurements with the ATLAS detector at LHC" hep-ex/0403021

[3] Etienvre, A. I.; Meyer, J. P.; Schwindling, J., "Top quark mass measurement in the lepton plus jets channel using full simulation" CERN-ATL-PHYS-INT-2005-002

[4] D.Brown, M.Frank, ALEPH internal note 92-135

[5] Correard, S; Kostyukhin, V; Lévêque, J; Rozanov, A; De Vivie de Régie, J B., "b-tagging with DC1 data" ATL-PHYS-2004-006

[6] Bentvelsen, S; Cobal, M, "Top studies for the Atlas detector commissioning" ATL-PHYS-PUB-2005-024 